\title{
Efficient Calculation of Power Bus Impedance using a Fast Algorithm Together with a Segmentation Method
}

\author{
Zhi Liang Wang* Non-member \\ Osami Wada** Member \\ Yoshitaka Toyota ${ }^{* *}$ Non-member \\ Ryuji Koga* $^{* *}$ Non-member
}

Power bus noise prediction in multilayer printed circuit boards requires an accurate estimation of the power bus impedance. We have previously developed a fast algorithm for efficiently and accurately calculating the impedance of a rectangular power bus structure, based on a cavity mode model. In this paper, we show that the fast algorithm can also be easily utilized for a right-angled triangular power bus structure. Together with a segmentation method, the fast algorithm can then be extended to calculate the impedances of more complicated power bus structures whose patterns consist of several segments of rectangles and/or rightangled triangles. Fast computation of the $S$-parameters and good agreement between the calculated results and measurements for various boards demonstrated the efficiency and accuracy of using the fast algorithm together with the segmentation method as a powerful technique to estimate the power bus impedance.

Keywords: power bus resonance, cavity-mode model, efficient calculation of power bus impedance, segmentation method

\section{Introduction}

Power bus resonances in multilayer printed circuit boards (PCBs) have recently attracted much attention, since the resonances not only cause radiated emission as EM interference, but also give rise to simultaneous switching noise as a signal integrity problem in highspeed digital circuits. Power bus noise suppression has thus become a major concern for EMC engineers engaged in high-speed PCB design. Efficient and accurate estimation of the power bus noise early in a board's design cycle is desirable to ensure the signal integrity and EMC of the product. The main aim of our work has been to develop a modeling tool suitable for this purpose.

Current multilayer PCBs often use entire solid powerreturn (ground) plane pairs for DC power distribution (a power bus). Though various modeling methods, such as the distributed lumped-element equivalent circuit model ${ }^{(1)(2)}$, partial element equivalent circuit (PEEC) approach $^{(3)}$, and numerical models based on the finite-element method ${ }^{(4)}$ and finite-difference timedomain method ${ }^{(5)}$ have been successfully applied to model power bus structures in PCBs, these models are relatively complex and require a significant amount of computing time to achieve accurate results.

In addition to the above-mentioned models, a full

* The Key Lab. of Wave Scattering and Remote Sensing Information of the Ministry of Education, Fudan University

Shanghai 200433, China

He was with the Faculty of Engineering, Okayama University, Japan

** The Faculty of Engineering, Okayama University

3-1-1, Tsushima-naka, Okayama 700-8530
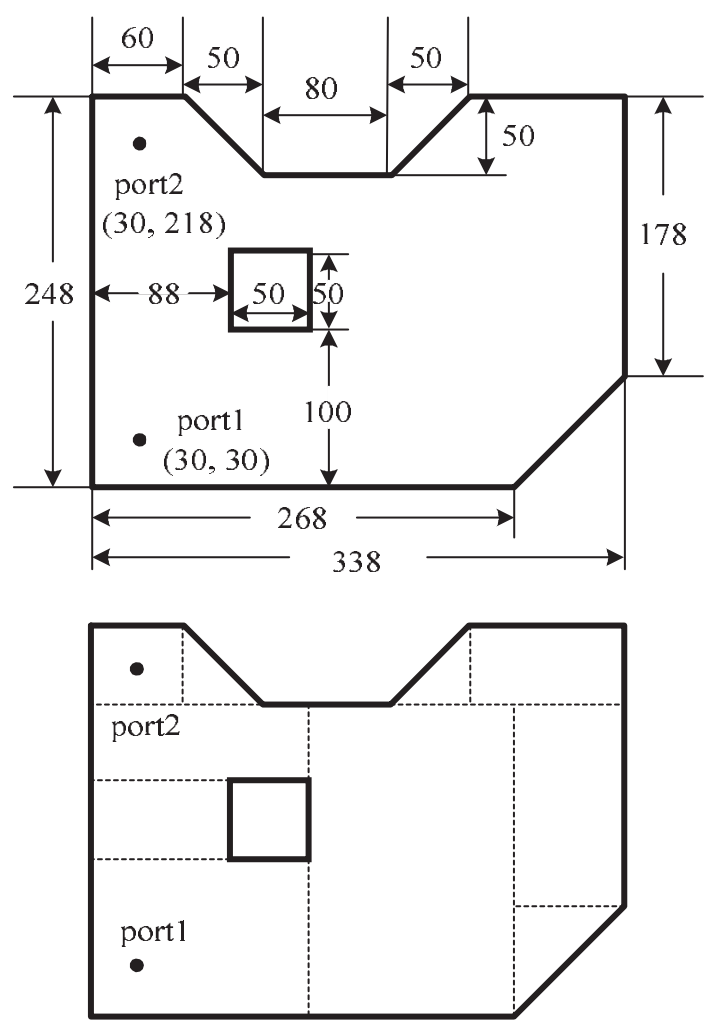

Fig. 1. Geometry of a power bus for simulation and measurement

cavity-mode model ${ }^{(6)(7)}$ has also been used to characterize the rectangular power bus structure as a planar 


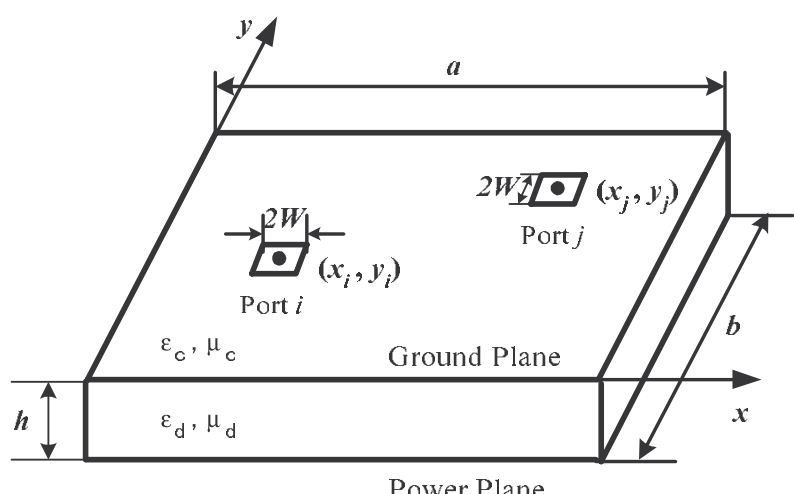

Power Plane

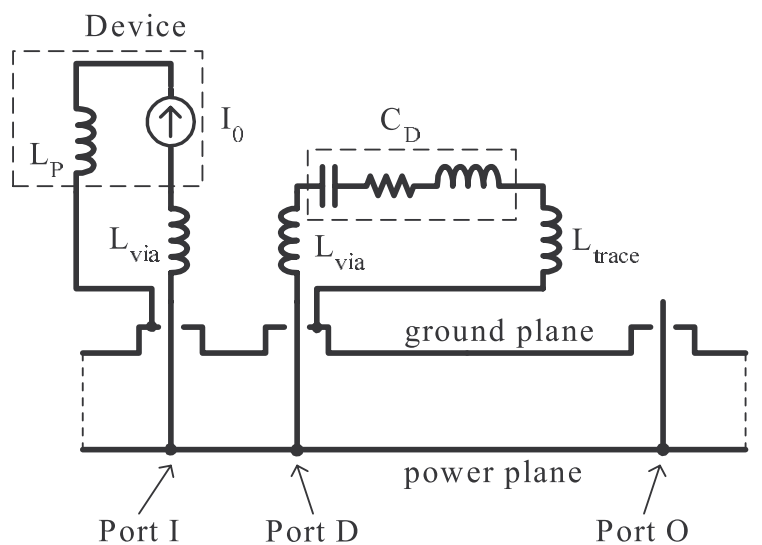

Fig. 2. Geometry of a rectangular power bus and its planar circuit model

multi-port microwave circuit ${ }^{(8)}$. Based on the cavitymode model, we have developed a fast algorithm for efficiently and accurately calculating the impedance of a rectangular power bus structure ${ }^{(9) \sim(11)}$. The fast algorithm is based on a closed-form expression for the impedance Z-matrix of a rectangular power bus structure; this expression was obtained by reducing the original double infinite series into a single infinite series under an approximation. The convergence of the single series is in further accelerated analytically. With the fast algorithm, using just a few terms has been sufficient to achieve good accuracy.

In this paper, we will show that the fast algorithm developed for a rectangular power bus structure can also be easily utilized for calculating the impedance of a rightangled triangular power bus structure ${ }^{(12)}$, according to the relation between the Green's functions for these two structures. Though the pattern of a power bus in a practical PCB is usually complicated, in many cases the pattern can be divided into several segments, which have simpler shapes, such as rectangles and triangles, as shown in Fig. 1. In addition, a slight difference in the circumference of the pattern does not significantly affect the resonance characteristics, so that the shape of the pattern may be approximated with only rectangles and/or triangles. We show that the fast algorithm together with a segmentation method ${ }^{(13) \sim(16)}$ described in Sec. 3 can then be applied to more complicated power bus structures whose patterns consist of several seg- ments of rectangles and/or right-angled triangles. Fast computation of the $S$-parameters and good agreement between the calculated results and measurements for various boards, shown in Sec. 4, have demonstrated the efficiency and accuracy of using the fast algorithm together with the segmentation method as a powerful technique to estimate the power bus impedance.

\section{Fast Algorithm using Cavity Modes}

The cavity-mode model is an analytical description of the impedance matrix (Z-parameters) of an unpopulated power bus structure (a bare board). The impedance matrix can be expressed in terms of the eigenfunctions and eigenvalues of the Helmholtz problem, by selecting a Green's function ${ }^{(8)}$ of the 2-D Helmholtz equation with the boundary condition of the second kind (the perfect magnetic conductor sidewalls), since most PCBs are electrically thin. Each “ mode" in the Z-parameter expression corresponds to a pole in the impedance. The full-mode representation of the Z-parameters of the power/ground plane structure is an infinite summation of modes, and results in an infinite numbers of poles.

2.1 A Rectangular Power Bus For a rectangular power bus structure with length $a$ and width $b$ (see Fig. 2), based on the Green's function of the 2-D Helmholtz equation which satisfies the boundary condition of the second kind on the four sidewalls of the dielectric layer, the Z-matrix elements can be obtained as ${ }^{(6) \sim(8)}$

$$
\begin{aligned}
Z_{i j}= & \sum_{m=0}^{\infty} \sum_{n=0}^{\infty} \frac{\mathrm{j} \omega \mu_{d} h}{a b} \frac{C_{m} C_{n}}{k_{x m}^{2}+k_{y n}^{2}-\kappa^{2}} \\
& \times \operatorname{sinc}^{2}\left(k_{x m} \mathrm{w}\right) \operatorname{sinc}^{2}\left(k_{y n} \mathrm{w}\right) \\
& \times \cos \left(k_{x m} x_{i}\right) \cos \left(k_{y n} y_{i}\right) \cos \left(k_{x m} x_{j}\right) \cos \left(k_{y n} y_{j}\right) \\
& \ldots \ldots \ldots \ldots \ldots \ldots(1)
\end{aligned}
$$

where $\operatorname{sinc}(x)=\sin (x) / x ; k_{x m}=m \pi / a, k_{y n}=n \pi / b$; $x_{i}, x_{j}, y_{i}$ and $y_{j}$ are the coordinates of the center of the $i$ th and $j$ th ports in the $x$ - and $y$-directions, respectively; $w$ is much less than the wavelengths of interest and represents the port half width (we assume for simplicity that the port sizes in the $x$ - and $y$-directions for the $i$ th and $j$ th ports are the same); $h$ is the dielectric thickness (spacing) between the power/ground planes; $\omega$ is the radian frequency; and $\mathrm{j}=\sqrt{-1}$. The constant $C_{m}=1$ if $m=0$, and $C_{m}=2$ if $m \neq 0$. Similarly, $C_{n}=1$ if $n=0$, and $C_{n}=2$ if $n \neq 0$.

The complex quantity $\kappa$ in the denominator of Eq. (1) is the transverse wavenumber $(\kappa=-\mathrm{j} \gamma, \gamma$ the transverse propagation constant) for the 2-D Helmholtz equation which reduces to the complex wavenumber $k_{d}$ of the dielectric if the conductors of the power/ground planes being considered to be perfect, as commonly treated in Ref. (6) (8). Howerver, to exactly predict the resonance properties of the power bus structure, it is necessary to take into account the effect of losses due to the power/ground plane conductors, dielectric and radiation. The radiation loss is usually small compared to the dielectric and copper losses in normal power bus 


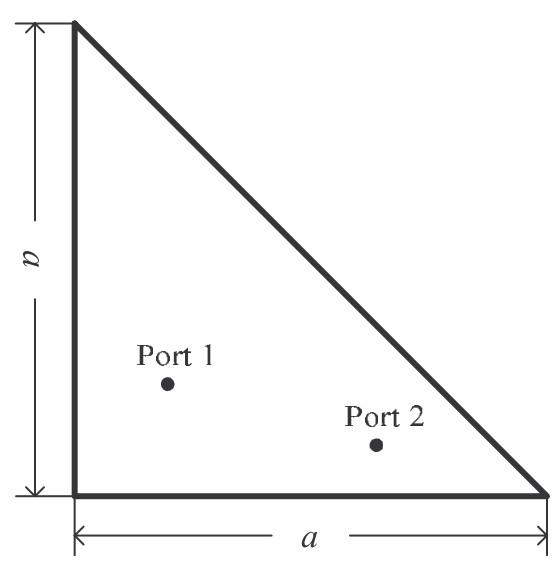

Fig. 3. Geometry of an equilateral right-angled triangular power bus

geometries, and can be neglected in the impedance calculation. The dielectric loss naturally appears in the imaginary part of the dielectric constant, while the conductor loss should be incorporated by considering the change in 2-D transverse wavenumber due to the finite conductivity. The model used to determine the transverse wavenumber $\kappa$ was described in Ref. (9), (10), and leads to the expression

$$
\kappa=\sqrt{\omega^{2} \mu_{d} \varepsilon_{d}-\mathrm{j} 2 \omega \varepsilon_{d} Z_{s} / h}
$$

where $\mu_{d}$ and $\varepsilon_{d}$ denote the permeability and permittivity of the dielectric; $Z_{\mathrm{s}}$ represents the surface impedance of the power/ground conductors.

As Fig. 2 shows, a board mounted with active devices or passive components can be considered a multi-port circuit network interconnected by the Z-matrix elements of the bare board. In principle, for a $P$-port circuit network, the number of Z-matrix elements that have to be calculated is $P(P+1) / 2$ for each frequency of interest. The computation of the double summation in Eq. (1) is obviously time consuming, so it is not practical if $P$ is large. In addition, the double series is extremely slow to converge for the self-input impedance because of the singularity of the Green's function as the field point approaches to the source point. Therefore, by using a summation formula of a Fourier series:

$$
\sum_{m=0}^{\infty} \frac{C_{m} \cos m x}{m^{2}-\alpha^{2}}=\frac{\pi}{\alpha} \frac{\cos (x-\pi) \alpha}{\sin \pi \alpha} \ldots \ldots \ldots \ldots
$$

the double series in Eq. (1) has been reduced into a much more rapidly converging single series. The resulting series is given as ${ }^{(9) \sim(11)}$

$$
\begin{aligned}
Z_{i j}^{(\mathrm{rec})}= & \sum_{n=0}^{\infty} \frac{\omega \mu_{d} h a}{\mathrm{j} 2 b} C_{n} \cos \left(k_{y n} y_{i}\right) \cos \left(k_{y n} y_{j}\right) \\
& \times \operatorname{sinc}^{2}\left(k_{y n} \mathrm{w}\right) \frac{\left[\cos \left(\alpha_{n} x_{-}\right)+\cos \left(\alpha_{n} x_{+}\right)\right]}{\alpha_{n} \sin \alpha_{n}}
\end{aligned}
$$

where $\alpha_{n}=a \sqrt{\kappa^{2}-k_{y n}^{2}}$, and $x_{ \pm}=1-\left(x_{i} \pm x_{j}\right) / a$. Notice that for reducing Eq. (1) into Eq. (4), we have made use of the approximation $\operatorname{sinc}^{2}\left(k_{x m} \mathbf{w}\right) \simeq 1(\mathbf{w} \rightarrow 0)$ in order to make use of the summation formula (3) for the index $m$ (if the summation is made for the index $n$, then the approximation should be $\left.\operatorname{sinc}^{2}\left(k_{y n} \mathbf{w}\right) \simeq 1\right)$. This approximation physically means that the two-dimensional port is now regarded as a one-dimensional port.

When the single series in Eq. (4) is used for calculating the self-input impedance $\left(Z_{i i}\right)$, it was found that for a small port dimension the convergence of the single series is still poor and thousands of terms must be computed to obtain good accuracy. We have developed a fast algorithm ${ }^{(11)}$ in which the convergence of the single series was in further accelerated analytically. With the fast algorithm, using just a few terms has been sufficient to achieve good accuracy. Moreover, we proposed two techniques to compensate for the errors between the selfinput impedance calculated using the double summation and that using the single summation; this error is due to the approximation involved in the process of reducing the double series to the single series. Therefore, the impedance of a rectangular power bus can be efficiently and accurately calculated with the fast algorithm.

2.2 A Right-angled Triangular Power Bus

For an equilateral right-angled triangular power bus structure with length $a$ shown in Fig. 3, the Green's function is given as ${ }^{(8)}$

$$
\begin{aligned}
& G_{i j}^{(\text {tri })}\left(x_{i}, y_{i}, x_{j}, y_{j}\right) \\
& =j \omega \mu_{d} d \sum_{m=0}^{\infty} \sum_{n=0}^{\infty} \frac{C_{m} C_{n} T\left(x_{i}, y_{i}\right) T\left(x_{j}, y_{j}\right)}{2\left[\left(m^{2}+n^{2}\right) \pi^{2}-a^{2} \kappa^{2}\right]} \\
& \ldots \ldots \ldots \ldots \ldots \ldots \ldots(5) \ldots \ldots \ldots \\
& T(x, y)=\cos \left(\frac{m \pi x}{a}\right) \cos \left(\frac{n \pi y}{a}\right) \\
& \quad+(-1)^{m+n} \cos \left(\frac{n \pi x}{a}\right) \cos \left(\frac{m \pi y}{a}\right)
\end{aligned}
$$$$
\ldots \ldots \ldots \ldots \ldots \cdots(6)
$$

Comparing the expression (5) with the Green's function of a square (a rectangle with $b=a$ )

$$
\begin{gathered}
G_{i j}^{(\mathrm{sq})}\left(x_{i}, y_{i}, x_{j}, y_{j}\right) \\
=\mathrm{j} \omega \mu_{d} d \sum_{m=0}^{\infty} \sum_{n=0}^{\infty} \frac{C_{m} C_{n} T_{0}\left(x_{i}, y_{i}\right) T_{0}\left(x_{j}, y_{j}\right)}{\left[\left(m^{2}+n^{2}\right) \pi^{2}-a^{2} \kappa^{2}\right]} \\
\ldots \ldots \ldots \ldots \ldots \ldots \ldots \ldots \\
T_{0}(x, y)=\cos \left(\frac{m \pi}{a} x\right) \cos \left(\frac{n \pi}{a} y\right) \ldots \ldots \ldots \ldots
\end{gathered}
$$

we then arrive at the relation

$$
\begin{aligned}
& G_{i j}^{(\mathrm{tri})}\left(x_{i}, y_{i}, x_{j}, y_{j}\right) \\
& \quad=G_{i j}^{(\mathrm{sq})}\left(x_{i}, y_{i}, x_{j}, y_{j}\right)+G_{i j}^{(\mathrm{sq})}\left(a-x_{i}, a-y_{i}, x_{j}, y_{j}\right) \\
& \ldots \ldots \ldots \ldots \ldots \ldots \ldots(9)
\end{aligned}
$$

This directly leads to an expression of the transfer impedance between two ports or the self-input impedance at a ports on a right-angled triangle can be expressed in terms of the corresponding one on a square 


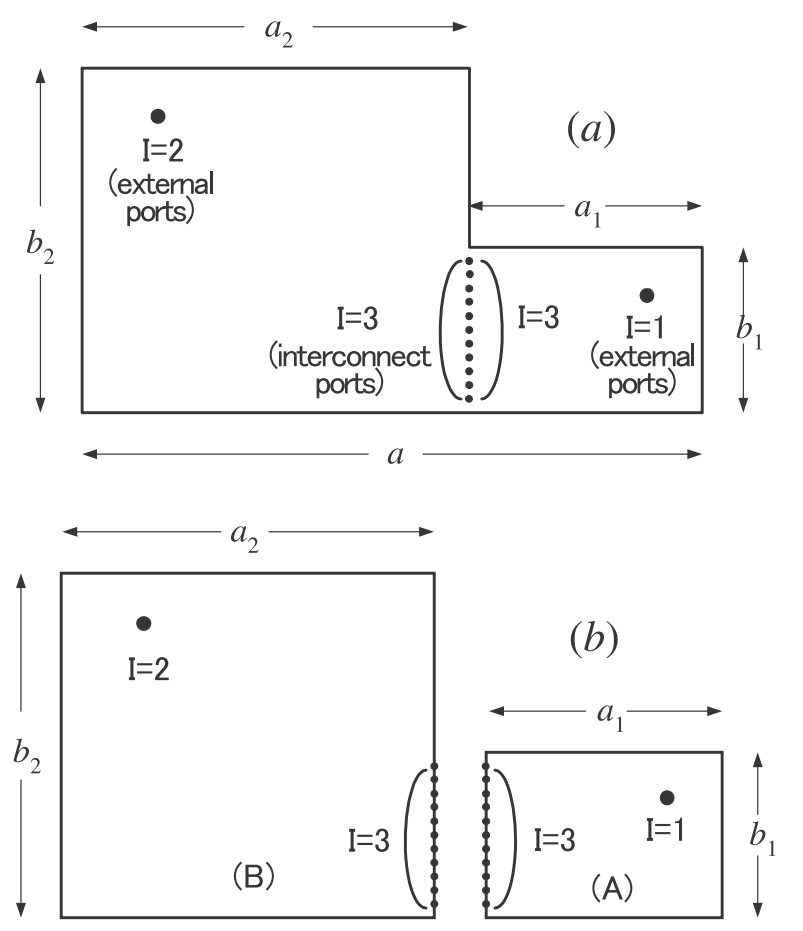

Fig. 4. Principle of the segmentation method. (a) An example of the power bus pattern to which the segmentation method can be applied, (b) Dividing into segments

(a rectangle with $b=a$ ) as ${ }^{(12)}$

$$
\begin{aligned}
& Z_{i j}^{(\mathrm{tri})}\left(x_{i}, y_{i}, x_{j}, y_{j}\right) \\
& \quad=Z_{i j}^{(\mathrm{rec})}\left(x_{i}, y_{i}, x_{j}, y_{j}\right)+Z_{i j}^{(\mathrm{rec})}\left(a-x_{i}, a-y_{i}, x_{j}, y_{j}\right) \\
& \ldots \ldots \ldots \ldots \ldots \ldots \ldots(10)
\end{aligned}
$$

Thus the Z-matrix elements of a right-angled triangular power bus $Z_{i j}^{\text {(tri) }}$ can also be efficiently and accurately calculated using the fast algorithm developed for the rectangular power bus structure.

\section{Segmentation Method}

The fast algorithm described above for the rectangular and triangular power bus geometries can be easily applied to those geometries resulting from the combination of rectangles and triangles, by using the segmentation method ${ }^{(13) \sim(15)}$ that was proposed many years ago for analyzing two-dimensional microwave planar circuits. This is illustrated i $\mathrm{n}$ a simple example in Fig. 4. The structure of Fig. 4(a) can be decomposed into the cascade of two segments in Fig. 4(b) for which the impedance matrices $\left[Z_{A}\right]$ and $\left[Z_{B}\right]$ can be computed with Eq. (1) or Eq. (4). By grouping the ports on the segments into the external ports (group $\mathrm{I}=1$ on Seg. (A) and group $\mathrm{I}=2$ on Seg. (B)) and the internal connected ports (group $\mathrm{I}=3$ ), the Z-matrices can then be written as

$$
\left[\begin{array}{l}
\vec{V}_{\mathrm{A} 1} \\
\vec{V}_{\mathrm{A} 3}
\end{array}\right]=\left[\begin{array}{ll}
{\left[Z_{\mathrm{A} 11}\right]} & {\left[Z_{\mathrm{A} 13}\right]} \\
{\left[Z_{\mathrm{A} 31}\right]} & {\left[Z_{\mathrm{A} 33}\right]}
\end{array}\right]\left[\begin{array}{c}
\vec{I}_{\mathrm{A} 1} \\
\vec{I}_{\mathrm{A} 3}
\end{array}\right] \cdots
$$

and

$$
\left[\begin{array}{l}
\vec{V}_{\mathrm{B} 2} \\
\vec{V}_{\mathrm{B} 3}
\end{array}\right]=\left[\begin{array}{ll}
{\left[Z_{\mathrm{B} 22}\right]} & {\left[Z_{\mathrm{B} 23}\right]} \\
{\left[Z_{\mathrm{B} 32}\right]} & {\left[Z_{\mathrm{B} 33}\right]}
\end{array}\right]\left[\begin{array}{c}
\vec{I}_{\mathrm{B} 2} \\
\vec{I}_{\mathrm{B} 3}
\end{array}\right]
$$

where $\vec{V}$ and $\vec{I}$ are the voltage and current vectors at the ports. Using the continuty conditions imposed by the interconnection for these vectors, that is

$$
\vec{V}_{\mathrm{A} 3}=\vec{V}_{\mathrm{B} 3}, \vec{I}_{\mathrm{A} 3}=-\vec{I}_{\mathrm{B} 3}
$$

the overall Z-matrix is then given as

$$
\left[\begin{array}{l}
\vec{V}_{\mathrm{A} 1} \\
\vec{V}_{\mathrm{B} 2}
\end{array}\right]=\left[\begin{array}{ll}
{\left[Z_{11}\right]} & {\left[Z_{12}\right]} \\
{\left[Z_{21}\right]} & {\left[Z_{22}\right]}
\end{array}\right]\left[\begin{array}{c}
\vec{I}_{\mathrm{A} 1} \\
\vec{I}_{\mathrm{B} 2}
\end{array}\right] \ldots \ldots
$$

where

$$
\begin{aligned}
& {\left[Z_{11}\right]=\left[Z_{\mathrm{A} 11}\right]-\left[Z_{\mathrm{A} 13}\right]\left[Y_{\mathrm{AB}}\right]\left[Z_{\mathrm{A} 31}\right]} \\
& {\left[Z_{12}\right]=\left[Z_{\mathrm{A} 13}\right]\left[Y_{\mathrm{AB}}\right]\left[Z_{\mathrm{B} 32}\right] \ldots \ldots \ldots} \\
& {\left[Z_{21}\right]=\left[Z_{\mathrm{B} 23}\right]\left[Y_{\mathrm{AB}}\right]\left[Z_{\mathrm{A} 31}\right] \ldots \ldots \ldots} \\
& {\left[Z_{22}\right]=\left[Z_{\mathrm{B} 22}\right]-\left[Z_{\mathrm{B} 23}\right]\left[Y_{\mathrm{AB}}\right]\left[Z_{\mathrm{B} 32}\right]}
\end{aligned}
$$

with

$$
\left[Y_{\mathrm{AB}}\right]=\left(\left[Z_{\mathrm{A} 33}\right]+\left[Z_{\mathrm{B} 33}\right]\right)^{-1}
$$

The description given here is an illustration of the segmentation method in principle. A simpler way for practical applications can be found in Ref. (17). In the segmentation method, the interconnection between the segments is discretized into a finite number of ports, in which continuous voltage and current distributions along the interconnection are approximated by stepped functions. The number of "virtual" ports used for the interconnection therefore influences significantly the accuracy of the segmentation method. Usually the number of "virtual" ports to be used increases as the frequency of interest goes higher. A detailed discussion on this topic was given in Ref. (16).

\section{Results and Discussion}

Numerical calculations have been performed for various power bus structures. The infinite series in both Eq. (1) and Eq. (4) has to be truncated in practical calculations. The maximum number (for both $n_{\max }$ and $m_{\max }$ in the double series and $n_{\max }$ in the single series) used in the calculation is denoted by $N$. The obtained results are considered to be the converged ones, when the relative error on the magnitudes of the impedances calculated by truncating at $N$ and at $N+1$, is less than $10^{-4}$. The power bus is commonly fed by a coaxial probe or interconnected to active devices and passive components through circular vias, so the shape of a port is circular in practice rather than square as assumed in the cavity-mode model. The equivalent half width of a square port can be determined as $w=(\pi / 4) r$ where $r$ is the radius of the corresponding circular port, assuming that their circumferences are equal to each other. In all of the following calculations, a value of $0.3 \mathrm{~mm}$ for the 

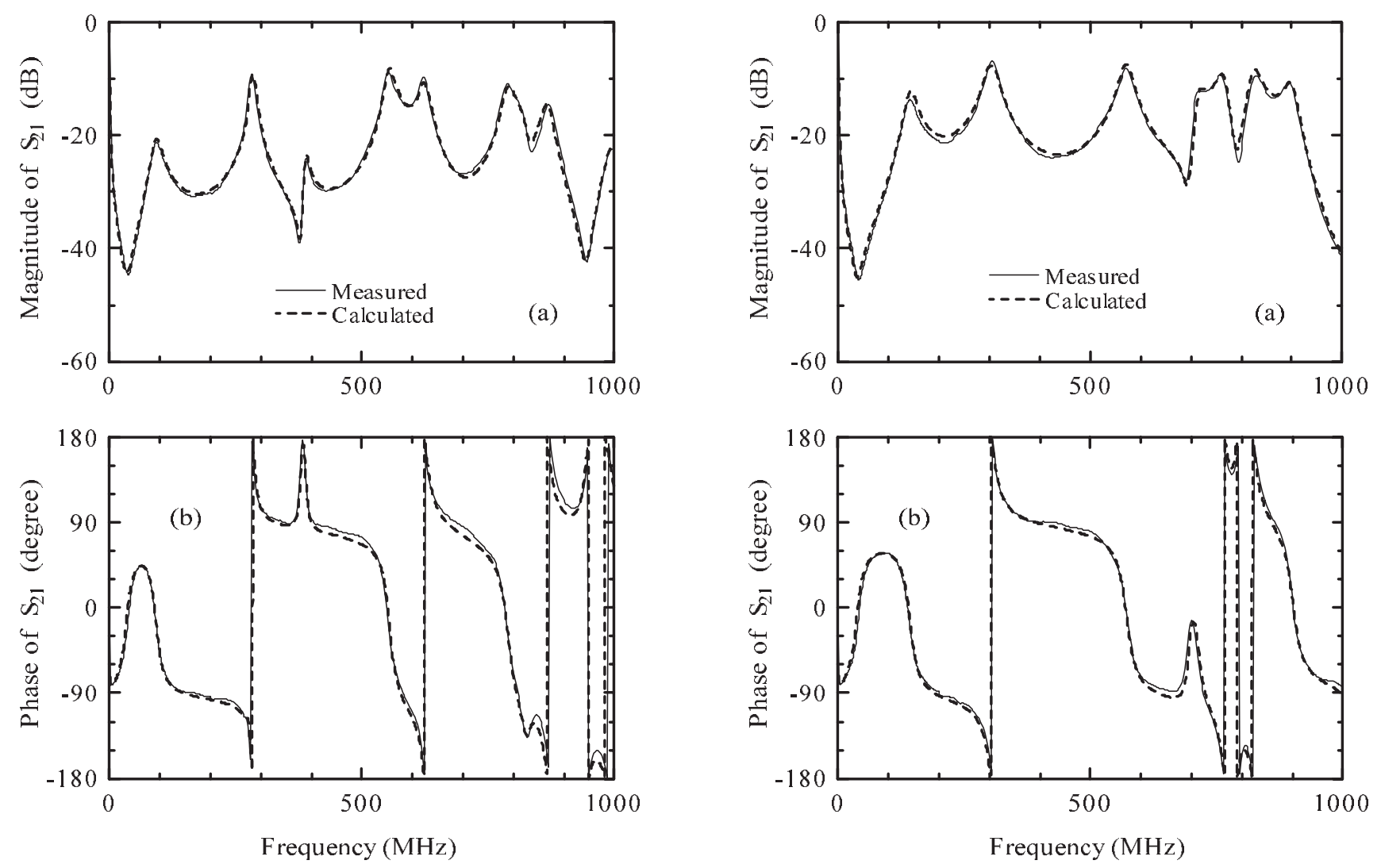

Fig. 5. Calculated and measured results for $S_{21}$ for Board A in Table 1: (a) Magnitude, and (b) Phase

Table 1. Dimensions for two test boards (mm)

\begin{tabular}{|l|c|c|}
\hline Name & Board A & Board B \\
\hline Board Sizes & $260 \times 260 \times 1.0$ & $260 \times 100 \times 1.0$ \\
\hline Feed Location & 10,110 & 10,30 \\
\hline Output Location & 250,110 & 250,30 \\
\hline Capacitor's & 50,130 & 50,50 \\
Locations & 130,130 & 130,50 \\
\hline
\end{tabular}

Table 2. Parameters of three thin boards

\begin{tabular}{|l|c|c|c|}
\hline Samples & $h(\mu \mathrm{m})$ & $\operatorname{Re}\left(\varepsilon_{r}\right)$ & $\tan \delta$ \\
\hline FR-4 & 110 & 3.92 & 0.021 \\
\hline BC2000 & 50 & 3.92 & 0.021 \\
\hline C-Ply & 13 & 21.5 & 0.0436 \\
\hline
\end{tabular}

half width $w$ of the practical ports has been used. Conductor for the power/ground planes is considered to be copper of conductivity $\sigma_{c}=5.92 \times 10^{7} \mathrm{~S} / \mathrm{m}$.

An evaluation on accuracy of the fast algorithm for the impedances of rectangular power bus structures has been carried out by comparisons between the calculated and measured $S$-parameters. Dimensions of the two test boards, each mounted with two decoupling capacitors, are summarized in Table 1. The two capacitors are the same, and their capacitance, parasitic inductance (equivalent series inductance (ESL) together with the inductance contributed by the connecting via and traces) and resistance (ESR) are rated to be $0.01 \mu \mathrm{F}, 1.2 \mathrm{nH}$ and $0.3 \Omega$, respectively. The relative permittivity of the
Fig. 6. Calculated and measured results for $S_{21}$ for Board B in Table 1: (a) Magnitude, and (b) Phase

dielectric (material: FR-4 glass epoxy) is assumed to be $\varepsilon_{r}=4.4-\mathrm{j} 0.088$ for both boards. The $S$-parameters can be obtained from the calculated impedances as described in Ref. (9). The calculated $S_{21}$ was compared to the measured data taken from Ref. (2), and plotted in Figs. 5 and 6 for Boards A and B, respectively. Very good agreement between the calculated results and measurements for both the magnitude and the phase demonstrated the accuracy of the fast algorithm.

In a recent study called NCMS Embedded Capacitance Projects, Hubing et.al. ${ }^{(18) \sim(20)}$ have confirmed that the board resonances can be completely damped by the conductive loss of the power/ground planes when the spacing between the planes (the dielectric thickness) is on the order of a skin depth in the conductor. To demonstrate the usefulness of Eq. (2) that incorporates the conductive loss into the impedance calculation, the input impedances for three thin boards are computed and plotted in Fig. 7. All three boards are the same in 76 $\mathrm{mm}$ length and $51 \mathrm{~mm}$ width, and fed at $(28 \mathrm{~mm}, 25.5$ $\mathrm{mm})$, but different in their dielectric permittivities and the spacing, as summarized in Table 2. The behavior of curves for the calculated input impedance seems to be quite in agreement with the measurements in Ref. (18), (19). Regardless of the dielectric loss, the resonant peaks in the input impedance disappeared due to the conductive loss as the spacing decreases to about $10 \mu \mathrm{m}$. The upward slope in the impedance curves is due to the connecting inductance of the SMA connector, and a value 


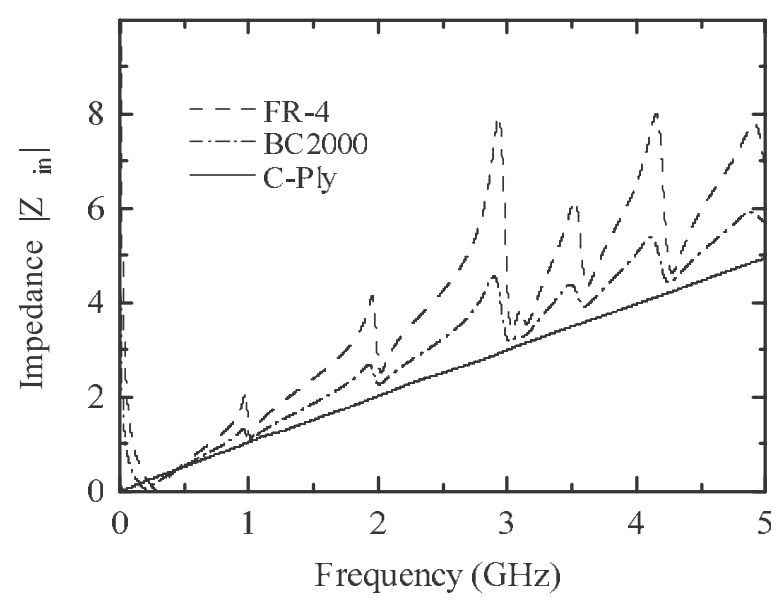

Fig. 7. Input impedance of three thin boards in Table 2 with different materials and spacing; All boards have $76 \mathrm{~mm}$ in length and $51 \mathrm{~mm}$ in width, and fed at $(28 \mathrm{~mm}, 25.5 \mathrm{~mm})$

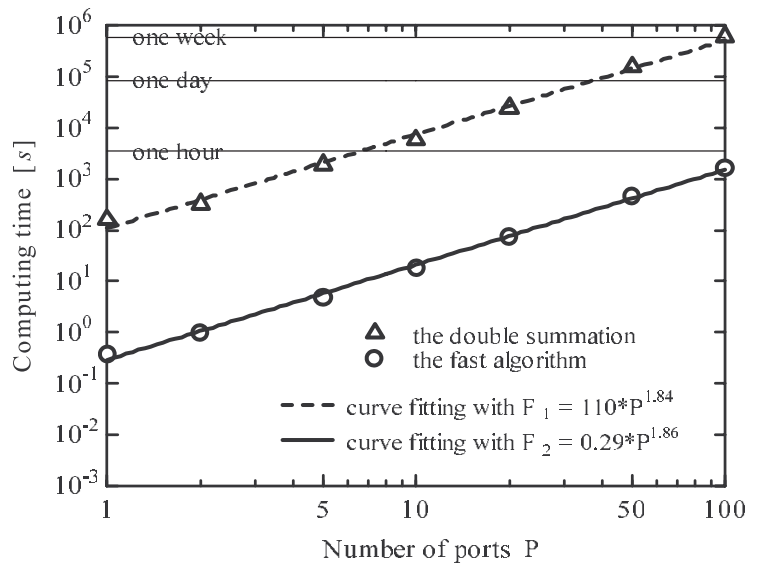

Fig. 8. Computing time as a function of the number of ports, when the double summation or the fast algorithm was used for calculation

of $150 \mathrm{pH}$ for this inductance has been used in the calculations.

To demonstrate the efficiency improvement provided by the fast algorithm, the computing time for obtaining the $S$-parameters over 3000 frequency points is plotted in Fig. 8 as a function of the number of ports, using the double summation in Eq. (1) and the fast algorithm developed in Ref.(11), respectively. The test board is a right-angled triangular board shown in Fig. 3 with $a=120 \mathrm{~mm}$. The board is fed at $(8 \mathrm{~mm}, 8 \mathrm{~mm})$, and mounted with $(P-1)$ decoupling capacitors where $P$ is the total number of the ports on the board. The capacitors are placed with $8 \mathrm{~mm}$ spacing each by each, and their capacitance, ESL and ESR are the same as given in the second paragraph of this section. For this example, the computing time of using the fast algorithm was roughly 380 times faster than that of using the double summation, regardless of the number of the ports used in the calculation. The calculation was done on an Alpha Station XP900 computer with $466 \mathrm{MHz}$ CPU.
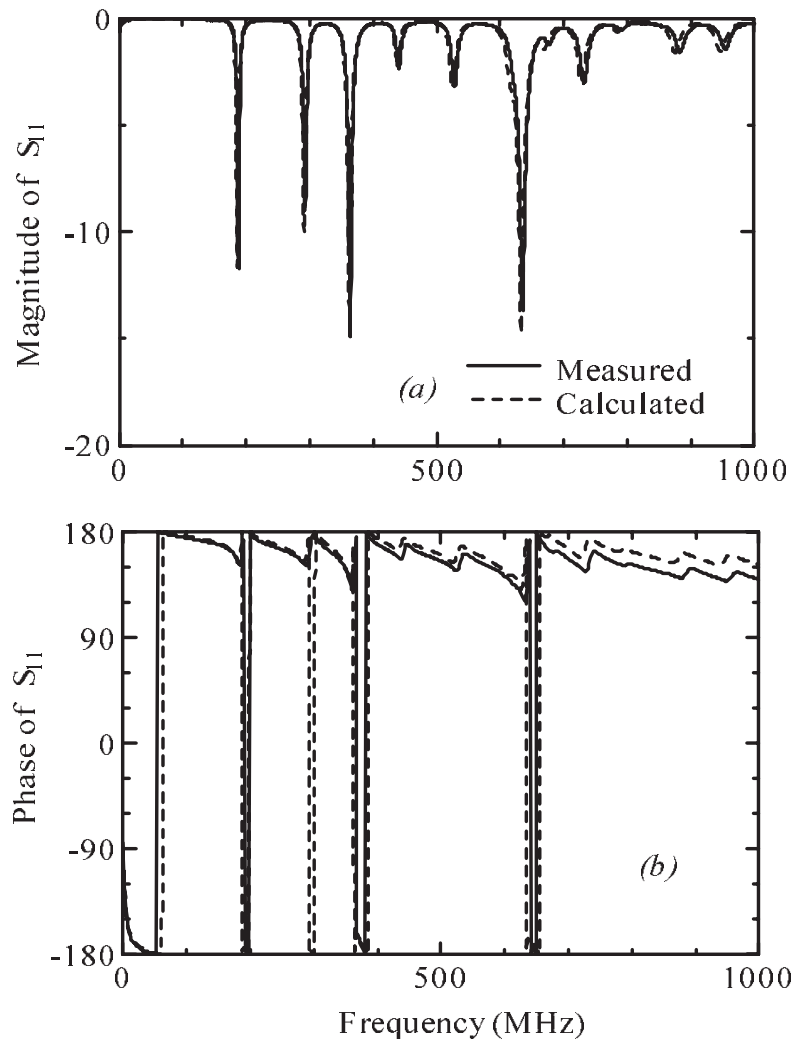

Fig. 9. Calculated and measured $S_{11}$ for the power bus geometry of Fig.1: (a) Magnitude, and (b) Phase
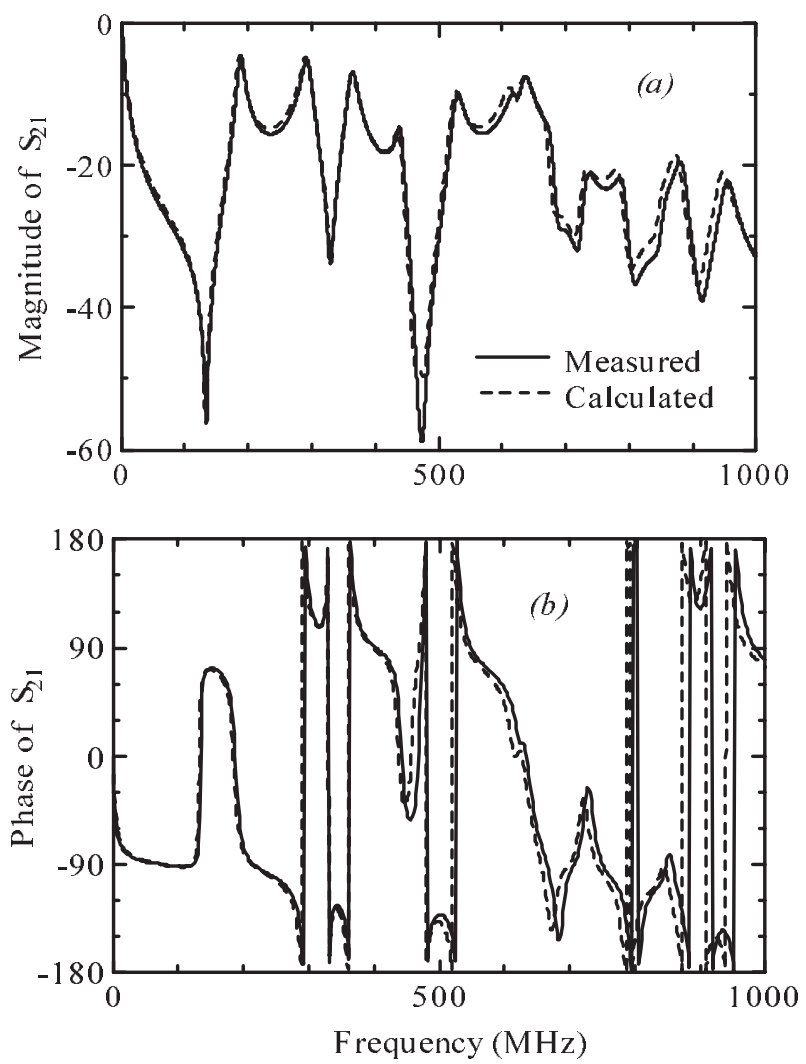

Fig. 10. Calculated and measured $S_{21}$ for the power bus geometry of Fig.1: (a) Magnitude, and (b) Phase 
The detailed discussion on the accuracy of the segmentation method can be found in Ref. (12), (16). As an example of using the fast algorithm together with the segmentation method, the calculated $S_{11}$ and $S_{21}$ for the power bus geometry of Fig. 1 are plotted and compared with measurements in Figs. 9 and 10. The calculated results agree well with the measured ones for both the magnitude and the phase. The computing time is about 3 minutes in a computer with dual $933 \mathrm{MHz}$ CPUs, for 1000 frequency points and 120 "virtual" ports used for interconnecting the segments. The "virtual" ports are placed along the dashed line interconnecting the segments, as shown in Fig. 1. Their total number is automatically determined by assuming that 15 "virtual" ports are needed for a section with a length of one wavelength in the dielectric at the highest frequency being considered ( $1 \mathrm{GHz}$ in the present case).

\section{Conclusion}

A fast algorithm developed for efficiently and accurately calculating the impedance of a rectangular power bus structure can be easily utilized for a right-angled triangular power bus structure. The fast algorithm together with a segmentation method has been extended to the case of more complicated power bus geometries whose patterns consist of several segments of rectangles and right-angled triangles. Fast computation of the $S$ parameters and good agreement between the calculated results and measurements for various boards demonstrated the efficiency and accuracy of using the fast algorithm together with the segmentation method as a powerful technique to estimate the power bus impedance.

\section{Acknowledgment}

We thank Dr. Harada of EMC Engineering Center, NEC Corporation of Japan for providing the measurement data of PCBs, and Prof. Todd H. Hubing of the University of Missouri-Rolla for helpful discussion.

This work was supported by the Project for the Reduction of Electromagnetic Noise, Research for the Future Program, Japan Society for the Promotion of Science (JSPS).

(Manuscript received Feb. 12, 2004, revised May 26, 2004)

\section{References}

(1) K. Lee and A. Barber: "Modeling and analysis of multichip module power supply planes", IEEE Trans. Comp. Packag. Manufact. Technol. B, Vol.18, pp.628-639 (1995-11)

(2) T. Harada: "A study on modeling and frequency characteristics of power-distribution planes in multilayer printed circuit boards responsible for radiated emission", Ph. D. Dissertation, The University of Electro-Communications, Japan (2000-3)

(3) B. Archambeault and A. Ruehli: "Electrical package modeling including voltage and ground reference planes using the partial element equivalent circuit (PEEC) method", Proc. 13th Int. Zurich Symp. Electromagnetic Compatibility, Zurich, Switzerland (1999-2)

(4) J. Fan, J.L. Drewniak, H. Shi, and J.L. Knighten: "DC powerbus modeling and design with a mixed-potential integralequation formulation and circuit extraction", IEEE Trans. Electromagn. Compat., Vol.43, pp.426-436 (2001-11)
(5) X. Ye, M.Y. Koledintseva, M. Li, and J.L. Drewniak: "DC power-bus design using FDTD modeling with dispersive media and surface mount technology components", IEEE Trans. Electromagn. Compat., Vol.43, pp.579-587 (2001-11)

(6) C.-T. Lei, R.W. Techentin, P.R. Hayees, D.J. Schwab, and B.K. Gilbert: "Wave model solution to the ground/power plane noise problem", IEEE Trans. Instrum. Meas., Vol.44, pp.300-303 (1995-4)

( 7 ) C.-T. Lei, R.W. Techentin, and B.K. Gilbert: "High-frequency characterization of power/ground-plane structures", IEEE Trans. Microwave Theory Tech., Vol.47, pp.562-569 (19995)

(8) J. Helszajn: Green's Function, Finite Elements and Microwave Planar Circuits, New York, John Wiley \& Sons (1996)

(9) Z.L. Wang, O. Wada, Y. Toyota, and R. Koga: "An improved closed-form expression for accurate and rapid calculation of power/ground plane impedance of multilayer PCBs", Proc. of Symposium on Electromagnetic Theory, EMT-00-68, Toyama, Japan (2000-10)

(10) Z.L. Wang, O. Wada, Y. Toyota, and R. Koga: "Reducing Q-factors of resonances in power/ground planes of multilayer PCBs by using resistive metal films", T. IEE Japan, Vol.121A, No.10, pp.928-932 (2001-10)

(11) Z.L. Wang, O. Wada, Y. Toyota, and R. Koga: "Convergence acceleration and accuracy improvement in power bus impedance calculation with a fast algorithm using cavity modes", IEEE Trans. Electromagn. Compat. (2005) (to be published)

(12) Z.L. Wang, O. Wada, Y. Toyota, and R. Koga: "Analysis of resonance characteristics of a power bus with rectangle and triangle elements in multilayer PCBs", Proc. 3rd Asia-Pacific Conference on Environmental Electromagnetics (CEEM'2003), pp.73-76, Hangzhou, China (2003-11)

(13) T. Okoshi, Y. Uehara, and T. Takeuchi: "The Segmentation Method-An Approach to the Analysis of Microwave Planar Circuits", IEEE Trans. Microwave Theory Tech., Vol.24, pp.662-668 (1976-10)

(14) R. Chadha and K.C. Gupta: "Segmentation Method Using Impedance Matrices for Analysis of Planar Microwave Circuits", IEEE Trans. Microwave Theory Tech., Vol.29, pp.71$74(1981-1)$

(15) R. Sorrentino: "Planar Circuits, Waveguide Models, and Segmentation Method", IEEE Trans. Microwave Theory Tech., Vol.33, pp.1057-1066 (1985-10)

(16) Z.L. Wang, O. Wada, Y. Toyota, and R. Koga: "Application of segmentation method to analysis of power/ground plane resonance in multilayer PCBs", Proc. 3rd Int. Symposium on Electromagnetic Compatibility, pp.775-778, Beijing, China (2002-5)

(17) Z.L. Wang, O. Wada, Y. Toyota, and R. Koga: "Modeling of gapped power bus structures for isolation using cavity modes and segmentation", IEEE Trans. Electromagn. Compat. (2005) (to be published)

(18) T.H. Hubing, M. Xu, J. Chen, J.L. Drewniak, T.P. Van Doren, and R.E. DUBroff: "Printed Circuit Board Power Bus Decoupling Using Embedded Capacitance", Proc. of the European Symposium on Electromagnetic Compatibility, pp.639642, Brugge (2000-9)

(19) T.H. Hubing, M. Xu, and J. Chen: "Electrical Model and Test Results for Embedded Capacitance Boards", Embedded Capacitance Project - Final Report, National Center for Manufacturing Sciences (NCMS), 3025 Boardwalk Ann Arbor, Michigan 48108-3266, USA (2000-3)

(20) M. Xu, T.H. Hubing, J. Chen, T.P. Van Doren, J.L. Drewniak, and R.E. DUBroff: "Power-Bus Decoupling with Embedded Capacitance in Printed Circuit Board Design", IEEE Trans. Electromagn. Compat., Vol.45, pp.22-30 (2003-2) 
Zhi Liang Wang (Non-member) was born in Zhejiang

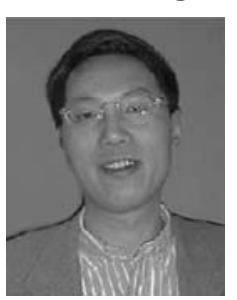
Province, China, on March 26, 1965. He received the B.E. and Ph.D. degrees in electrical engineering from Nanjing University of Posts and Telecommunications in 1983, and University of Electronic Science and Technology of China (UESTC, Chengdu, China) in 1988, respectively. From 1988 to 1994, he was with UESTC as a Lecturer and an Associate Professor. From 1994 to 1997, he was with Kyoto University as a Visiting Scholar and a part-time Lecturer. From 1997-2000, he was with the Communications Research Laboratory in Tokyo as a Research Associate. From April 2000-March 2004, he was with Okayama University as a Research Associate working on EMC problems. He is currently a Professor at the Key Laboratory of Wave Scattering and Remote Sensing Information of the Ministry of Education, Fudan University, Shanghai, China. His research fields include electromagnetic theory, optical fiber science, microwave power transmission, integrated optical waveguide, wave propagation and scattering in random media and from rough surfaces, as well as electromagnetic compatibility. Dr. Wang is a member of IEICE of Japan, and IEEE.

Osami Wada (Member) was born in Osaka, Japan, on July

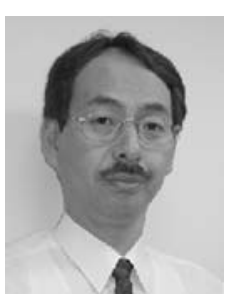
3, 1957. He received the B.E., M.E. and Dr.E. degrees in electronics from Kyoto University, Japan, in 1981, 1983, and 1987, respectively. In the university he was engaged in research on transmission system and quasioptical antenna systems for high-power millimeter waves. Since 1988, he has been with the Faculty of Engineering, Okayama University. He is currently an Associate Professor of the Department of Communication Network Engineering. He has engaged in study of electromagnetic compatibility of digital circuit boards and systems, development of optical integrated circuits, control of laser beam profile, and atmospheric monitoring with lasers. Dr. Wada is a member of IEICE (the Institute of Electronics, Information and Communication Engineers), IEE of Japan, Japan Institute of Electronics Packaging, the Japan Society of Applied Physics, Optical Society of Japan and IEEE.
Yoshitaka Toyota (Non-member) was born in Okayama,

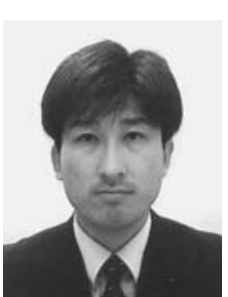

Japan, on September 17, 1968. He received the B.E. and M.E. degrees from Okayama University, Japan, in 1991 and 1993, respectively, and the D.E. degree from Kyoto University, Japan, in 1996. From 1996 to 1998, he was with Yokogawa Electric Co., Ltd. Since 1998, he has been a research associate in Okayama University. His recent research interests are optical integrated circuits, measurement of atmosphere with lasers, and EMC design for high-speed digital systems. Dr. Toyota is a member of the Japan Society of Applied Physics (JSAP) and the Institute of Electronics, Information and Communication Engineers (IEICE).

Ryuji Koga (Non-member) was born in Tokyo, Japan, on

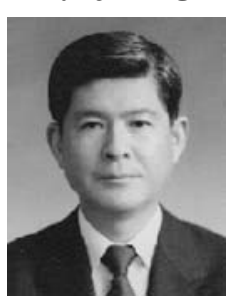
January 1, 1945. He received the B.E., M.E., and Dr. E. in Electrical Engineering from Kyoto University, Japan, in 1967, 1969 and 1975, respectively. From 1972 to 1976 he was with the Atomic Energy Institute, Kyoto University. Then he moved to the Department of Electronics, Okayama University, as a Lecturer. He is now a Professor of the Department of Communication Network Engineering, Faculty of Engineering, Okayama University. Dr. Koga is a member of the Institute of Electronics, Information and Communication Engineers and presently is a Vice Chairman of Electromagnetic Compatibility technical (EMCJ) group of Communications Society, Institute of Electronics, Information and Communication Engineers. 